\title{
Field line wandering and perpendicular scattering of charged particles in Alfvénic slab turbulence
}

\author{
A. Shalchi, R. C. Tautz, and R. Schlickeiser \\ Institut für Theoretische Physik, Lehrstuhl IV: Weltraum- und Astrophysik, Ruhr-Universität Bochum, 44780 Bochum, Germany \\ e-mail: andreasm4@yahoo.com
}

Received 7 June 2007 / Accepted 24 August 2007

\section{ABSTRACT}

\begin{abstract}
We describe analytically the magnetic field line random walk in Alfvénic slab turbulence. This result can easily be combined with a compound transport model to describe analytically the perpendicular scattering of charged cosmic rays. We demonstrate that the well known subdiffusive solution for magnetostatic turbulence is only valid for shorter time scales. For longer times, perpendicular diffusion of charged test-particles is recovered due to wave propagation effects. For low energy cosmic rays, this new result could be important for understanding heliospheric observations.
\end{abstract}

Key words. waves - turbulence - diffusion

\section{Introduction}

Understanding turbulence and particle transport in turbulent magnetic fields is an issue of major importance in space physics and astrophysics. It has been demonstrated in several articles that stochastic wandering of magnetic field lines directly influences the transport of charged cosmic rays (e.g., Kóta \& Jokipii 2000; Webb et al. 2006; Shalchi \& Kourakis 2007b). Several theories have been developed to describe field line random walk (FLRW) analytically.

The classic work of Jokipii (1966), for instance, employed a quasilinear approach for FLRW. To achieve a more reliable and general description of field line wandering, Matthaeus et al. (1995) developed a non-perturbative statistical approach by combining certain assumptions about the properties of the field lines (e.g., Gaussian statistics) with a diffusion model. More precisely, in the Matthaeus et al. theory of field line wandering, it is explicitly assumed that field line wandering behaves diffusively.

An improved theory for FLRW, which is a generalization of the theory of Matthaeus et al., was recently developed by Shalchi \& Kourakis (2007a). By explicitly assuming diffusive behavior of the field lines, the Matthaeus et al. theory can be obtained from the Shalchi \& Kourakis (2007a) approach as a special limit. However, the improved theory of Shalchi \& Kourakis (2007a) provides a superdiffusive behavior of FLRW if the socalled slab/2D composite model for the turbulence is applied. As shown in Shalchi \& Kourakis (2007b), this superdiffusive behavior of field line wandering leads to subdiffusion of charged cosmic rays perpendicular to the mean magnetic field. This nondiffusivity contradicts the assumption of classical diffusion that has been applied in previous cosmic ray transport studies. A possible reason for this non-diffusivity could be the assumption of magnetostatic turbulence, which has always been used in previous studies of FLRW.

We explore FLRW and charged particle transport in (undamped) Alfvénic slab turbulence. In Sect. 2, we discuss several models for dynamical turbulence and in Sect. 3, we describe the FLRW for Alfvénic slab turbulence analytically. In Sect. 4, we employ a compound transport model to describe charged particle transport. A comparison with test-particle simulations is presented in Sect. 5, and in Sect. 6 we investigate perpendicular transport in non-diffusive cases of FLRW. Section 7 summarizes our new results.

\section{Dynamical turbulence}

The key input into the theoretical description of FLRW is the so-called magnetic correlation tensor, whose components are

$R_{i j}\left(\boldsymbol{x}, \boldsymbol{x}_{0}, t, t_{0}\right)=\left\langle\delta B_{i}(\boldsymbol{x}, t) \delta B_{j}^{\star}\left(\boldsymbol{x}_{0}, t_{0}\right)\right\rangle$.

In Fourier space, its components have the form

$$
\begin{aligned}
P_{i j}(\boldsymbol{k}, t) & =\left\langle\delta B_{i}(\boldsymbol{k}, t) \delta B_{j}^{\star}(\boldsymbol{k}, 0)\right\rangle \\
& =\int \mathrm{d}^{3} k\left\langle\delta B_{i}(\boldsymbol{x}, t) \delta B_{j}^{\star}(0,0)\right\rangle \mathrm{e}^{-i \boldsymbol{k} \cdot \boldsymbol{x}},
\end{aligned}
$$

where we used $t_{0}=0, \boldsymbol{x}_{0}=0$, and the assumption of homogeneous turbulence

$\left\langle\delta B_{i}(\boldsymbol{k}, t) \delta B_{j}^{\star}\left(\boldsymbol{k}^{\prime}, 0\right)\right\rangle=P_{i j}(\boldsymbol{k}, t) \delta\left(\boldsymbol{k}-\boldsymbol{k}^{\prime}\right)$.

If we assume the same temporal behavior of all tensor components, we have

$P_{l m}(\boldsymbol{k}, t)=P_{l m}(\boldsymbol{k}) \Gamma(\boldsymbol{k}, t)$,

where we used the so-called dynamical correlation function $\Gamma(\boldsymbol{k}, t)$ and the magnetostatic correlation tensor $P_{l m}(\boldsymbol{k})$.

To date, two principal models describe the dynamics of the turbulent magnetic fields and the consequent the dynamical correlation function:

1. Dynamical turbulence: One of the first groups to discuss particle transport in dynamical turbulence was 
Bieber et al. (1994). In their article, the authors proposed two models for the dynamical correlation function:

$$
\begin{array}{ll}
\Gamma_{\mathrm{DT}}(\boldsymbol{k}, t)=\mathrm{e}^{-t / t_{\mathrm{c}}} & (\text { DT-model }) \\
\Gamma_{\mathrm{RS}}(\boldsymbol{k}, t)=\mathrm{e}^{-\left(t / t_{\mathrm{c}}\right)^{2}} & (\text { RS-model })
\end{array}
$$

with the correlation time scale $t_{\mathrm{c}}$. In the damping model of dynamical turbulence (DT model), the dynamical correlation function has an exponential form, whereas in the random sweeping model (RS model), $\Gamma(\boldsymbol{k}, t)$ has a Gaussian form. Bieber et al. (1994) estimated the correlation time as

$t_{\mathrm{c}}=\alpha v_{\mathrm{A}}|\boldsymbol{k}|$.

Here, $v_{\mathrm{A}}$ is the Alfvén speed and $\alpha$ is a parameter that allows the strength of the dynamical effects to be adjusted, ranging from $\alpha=0$ (magnetostatic turbulence) to $\alpha=1$ (strongly dynamical turbulence). Bieber et al. (1994) also suggested that the parameter $\alpha$ could be interpreted as $\delta B / B_{0}$. In this case, the correlation time scale $t_{\mathrm{c}}$ becomes comparable to the eddy turnover time. Also, decorrelation effects related to plasma waves (e.g. Schlickeiser \& Achatz 1993) can be achieved by expressing $\alpha$ through parameters such as the plasma $\beta$ (for a definition see also Schlickeiser \& Achatz 1993).

2. Plasma wave turbulence: Another prominent model is the plasma wave model which is discussed in Schlickeiser (2002). In this model, the dynamical correlation function has the form

$$
\Gamma_{\mathrm{PW}}(\boldsymbol{k}, t)=\mathrm{e}^{\mathrm{i} \omega t-\gamma t} .
$$

Here, $\omega$ is the plasma wave dispersion relation, whereas $\gamma$ describes plasma wave damping. Often, undamped plasma waves are considered, where $\Gamma_{\mathrm{PW}}(\boldsymbol{k}, t)=\mathrm{e}^{\mathrm{i} \omega t}$, and where the dynamical correlation function is a purely oscillating function. Prominent examples for different plasma waves are shear Alfvén waves, where $\omega= \pm v_{\mathrm{A}} k_{\|}$, and fast magnetosonic waves, where $\omega=v_{\mathrm{A}} k$.

\section{Field line random walk in Alfvénic slab turbulence}

In this section, we discuss the form of the field line mean square deviation (MSD) in a system which consists of a uniform mean magnetic field $\left(\boldsymbol{B}_{0}=B_{0} \boldsymbol{e}_{z}\right)$ and a turbulent component $\left(\delta B_{i}\right)$. We discuss well known results obtained for magnetostatic slab turbulence and investigate FLRW in Alfvénic turbulence.

\subsection{The magnetostatic slab model}

In the slab model, we assume that the turbulent magnetic field depends only on the coordinate parallel to the mean field $\boldsymbol{B}_{0}$ :

$\boldsymbol{B}(\boldsymbol{x})=B_{0} \boldsymbol{e}_{z}+\delta \boldsymbol{B}(z)$

and the $x x$ component of the correlation tensor has the form

$P_{x x}^{\text {slab }}(\boldsymbol{k})=g^{\text {slab }}\left(k_{\|}\right) \frac{\delta\left(k_{\perp}\right)}{k_{\perp}}$.

In several previous studies (e.g., Bieber et al. 1994), the form

$g^{\text {slab }}\left(k_{\|}\right)=\frac{C(v)}{2 \pi} l_{\text {slab }} \delta B_{\text {slab }}^{2}\left(1+k_{\|}^{2} l_{\text {slab }}^{2}\right)^{-v}$ for the slab wave spectrum $g^{\text {slab }}\left(k_{\|}\right)$has been used. Here, we used the slab bend-over scale $l_{\text {slab }}$, the strength of the turbulent field $\delta B_{\text {slab }}^{2}$, the inertial range spectral index $2 v$, and the function

$C(v)=\frac{1}{2 \sqrt{\pi}} \frac{\Gamma(v)}{\Gamma(v-1 / 2)}$.

As shown in several previous papers (e.g., Shalchi \& Kourakis 2007a), the field line MSD can be calculated exactly for pure slab geometry. For standard forms of the wave spectrum (e.g., Eq. (10)), we find a classic diffusive result (Jokipii 1966; Matthaeus et al. 1995):

$\left\langle(\Delta x)^{2}\right\rangle_{\mathrm{FL}}=2 \kappa_{\mathrm{FL}}|z|$

where the field line diffusion coefficient is defined as

$\kappa_{\mathrm{FL}}=\pi C(v) l_{\text {slab }} \frac{\delta B_{\text {slab }}^{2}}{B_{0}^{2}}$.

We then replace of the magnetostatic model by an undamped Alfvénic model.

\subsection{Alfvénic slab turbulence}

In this paragraph we will assume that the turbulence can be represented by undamped parallel propagating Shear Alfvén waves. In this case, the wave propagation velocity is the Alfvén speed $v_{\mathrm{A}}$. First, we transform to the rest frame of the wave, $K S^{\prime}$, where the magnetostatic solution for field line wandering can be used

$\left\langle(\Delta x)^{2}\right\rangle_{\mathrm{FL}}=2 \kappa_{\mathrm{FL}}\left|z^{\prime}\right|$.

By applying the Lorentz transformation,

$z^{\prime}=\frac{z \pm v_{\mathrm{A}} t}{\sqrt{1-\left(v_{\mathrm{A}} / c\right)^{2}}}$

we can describe FLRW in the laboratory system of coordinates $K S$. Here, " \pm " stands for the two possible plasma wave propagation directions. Because $v_{\mathrm{A}} \ll c$, we can approximately use the Galilei transformation

$z^{\prime} \approx z \pm v_{\mathrm{A}} t$

By substituting Eqs. (16) into (14), we find for the field line MSD

$\left\langle(\Delta x)^{2}\right\rangle_{\mathrm{FL}}=2 \kappa_{\mathrm{FL}}\left|z \pm v_{\mathrm{A}} t\right|$,

which is now a function of space and time. By setting $v_{\mathrm{A}}=0$, we can easily recover the static result of Eq. (12).

\section{Compound transport of charged particles}

A simple but reliable method for describing charged particle scattering in the direction perpendicular to the mean field $\boldsymbol{B}_{0}$ is a compound transport model, which is given by the equation

$\left\langle(\Delta x)^{2}\right\rangle_{\mathrm{P}}(t)=\int_{-\infty}^{+\infty} \mathrm{d} z\left\langle(\Delta x)^{2}\right\rangle_{\mathrm{FL}}(z, t) f_{\|}(z, t)$.

Here, we have defined the MSD of the particle displacement in the perpendicular direction, $\left\langle(\Delta x(t))^{2}\right\rangle_{\mathrm{P}}(t)$, the MSD of the field lines, $\left\langle(\Delta x(z))^{2}\right\rangle_{\mathrm{FL}}(z, t)$, and the parallel particle distribution function $f_{\|}(z, t)$. Equation (18) corresponds to the assumption that the particles follow magnetic field lines. If this assumption is true, we can set the field line MSD equal to the 
perpendicular MSD of the charged particle $\left\langle(\Delta x(t))^{2}\right\rangle_{\mathrm{P}}(t)=$ $\left\langle(\Delta x(z))^{2}\right\rangle_{\mathrm{FL}}(z(t), t)$, where $z(t)$ is the (time-dependent) parallel position of the particles. Since the particles also experience parallel scattering, $z(t)$ is a randomized paramterer, and Eq. (18) follows directly.

Equation (18) can also be obtained from the ChapmanKolmogorov equation (e.g., Webb et al. 2006), which has the form

$f_{\perp}(x, t)=\int_{-\infty}^{+\infty} \mathrm{d} z f_{\mathrm{FL}}(x, z) f_{\mathrm{P}}(z, t)$

with the particle distribution in the perpendicular direction $f_{\perp}(x, t)$ and the field line distribution function $f_{\mathrm{FL}}(x, z)$. As shown in Webb et al. (2006, Eq. (22)), Eq. (18) can be deduced from Eq. (19) by calculating the second moment of $f_{\perp}(x, t)$. Here, we assume that Eq. (18) is still valid in the case of a timedependent field line $\operatorname{MSD}\left\langle(\Delta x)^{2}\right\rangle_{\mathrm{FL}}(z, t)$, which is a reasonable assumption.

To proceed, we assume diffusive parallel propagation of the charged particles. In this case, and by assuming sharp initial conditions, the Gaussian function

$f_{\|, \mathrm{G}}(z, t)=\frac{1}{\sqrt{4 \pi t \kappa_{\|}}} \mathrm{e}^{-z^{2} /\left(4 t \kappa_{\|}\right)}$

solves the diffusion equation. We used the parallel diffusion coefficient $\kappa_{\|}$. For Alfvénic slab turbulence, Eq. (18) thus becomes

$\left\langle(\Delta x)^{2}\right\rangle_{\mathrm{P}}(t)=\frac{2 \kappa_{\mathrm{FL}}}{\sqrt{4 \pi t \kappa_{\|}}} \int_{-\infty}^{+\infty} \mathrm{d} z\left|z \pm v_{\mathrm{A}} t\right| \mathrm{e}^{-z^{2} /\left(4 t \kappa_{\|}\right)}$.

This integral can be written as

$$
\begin{aligned}
\left\langle(\Delta x)^{2}\right\rangle_{\mathrm{P}}(t)= & \frac{-2 \kappa_{\mathrm{FL}}}{\sqrt{4 \pi t \kappa_{\|}}} \\
& \times\left[\int_{-\infty}^{\mp v_{\mathrm{A}} t} \mathrm{~d} z\left(z \pm v_{\mathrm{A}} t\right) \mathrm{e}^{-z^{2} /\left(4 t \kappa_{\|}\right)}\right. \\
& \left.-\int_{\mp v_{\mathrm{A}} t}^{\infty} \mathrm{d} z\left(z \pm v_{\mathrm{A}} t\right) \mathrm{e}^{-z^{2} /\left(4 t \kappa_{\|}\right)}\right] .
\end{aligned}
$$

To proceed, we apply Gradshteyn \& Ryzhik (2000)

$$
\begin{aligned}
\int_{-\infty}^{u} \mathrm{~d} z z \mathrm{e}^{-\gamma^{2} z^{2}} & =\frac{-1}{2 \gamma^{2}} \mathrm{e}^{-\gamma^{2} u^{2}} \\
\int_{-\infty}^{u} \mathrm{~d} z \mathrm{e}^{-\gamma^{2} z^{2}} & =\frac{\sqrt{\pi}}{2 \gamma}[1+\operatorname{erf}(\gamma u)] \\
\int_{u}^{\infty} \mathrm{d} z z \mathrm{e}^{-\gamma^{2} z^{2}} & =\frac{1}{2 \gamma^{2}} \mathrm{e}^{-\gamma^{2} u^{2}} \\
\int_{u}^{\infty} \mathrm{d} z \mathrm{e}^{-\gamma^{2} z^{2}} & =\frac{\sqrt{\pi}}{2 \gamma}[1-\operatorname{erf}(\gamma u)]
\end{aligned}
$$

in terms of the error function erf $(z)$. By additionally applying

$\operatorname{erf}(-z)=-\operatorname{erf}(z)$,

Eq. (22) becomes

$\left\langle(\Delta x)^{2}\right\rangle_{\mathrm{P}}(t)=2 \kappa_{\mathrm{FL}}\left[\sqrt{\frac{4 \kappa_{\|} t}{\pi}} \mathrm{e}^{-\xi^{2}}+v_{\mathrm{A}} t \operatorname{erf}(\xi)\right]$,

where we used the parameter

$\xi=\frac{v_{\mathrm{A}}}{2} \sqrt{\frac{t}{\kappa_{\|}}}$.
Equation (25) is the exact result for the particle MSD in Alfvénic slab turbulence. In the following, we consider two asymptotic limits to simplify Eq. (25).

\subsection{The limit $\xi \ll 1$}

By introducing the diffusive time scale

$t_{\text {diffusive }}=\frac{\kappa_{\|}}{v^{2}}$

the parameter $\xi$ can be written as

$\xi=\frac{1}{2} \frac{v_{A}}{v} \sqrt{\frac{t}{t_{\text {diffusive }}}}$.

Since we take the particles to be diffusive (see Eq. (20)) we cannot consider regimes where $t \approx t_{\text {diffusive }}$ or $t<t_{\text {diffusive }}$. However, in most cases the ratio $v_{\mathrm{A}} / v$ is a very small number. Thus, the limit $\xi \ll 1$ is a physically reasonable limit, which is valid if the restriction

$t_{\text {diffusive }} \ll t \ll 4\left(\frac{v_{\mathrm{A}}}{v}\right)^{2} t_{\text {diffusive }}$

holds.

In the case considered here, we can use (Abramowitz \& Stegun 1974)

$$
\mathrm{e}^{-\xi^{2}} \approx 1
$$

$\operatorname{erf}(\xi) \approx \frac{2}{\sqrt{\pi}} \xi$

to deduce asymptotically

$$
\begin{aligned}
\left\langle(\Delta x)^{2}\right\rangle_{\mathrm{P}}(t) & \approx 4 \kappa_{\mathrm{FL}} \sqrt{\frac{\kappa_{\|} t}{\pi}}\left(1+\xi^{2}\right) \\
& \approx 4 \kappa_{\mathrm{FL}} \sqrt{\frac{\kappa_{\|} t}{\pi}} .
\end{aligned}
$$

This result corresponds to the magnetostatic limit obtained in previous articles (e.g., Webb et al. 2006; Shalchi \& Kourakis 2007b). Equation (31b) is the exact result for magnetostatic turbulence $\left(v_{\mathrm{A}} \rightarrow 0\right)$. Obviously, for time scales that satisfy the constraint $\xi \ll 1$, we find a subdiffusive behavior of perpendicular scattering of charged particles $\left(\left\langle(\Delta x)^{2}\right\rangle_{\mathrm{P}}(t) \propto \sqrt{t}\right)$.

\subsection{The limit $\xi \gg 1$}

In this case, we can use Abramowitz \& Stegun (1974)

$$
\begin{aligned}
\mathrm{e}^{-\xi^{2}} & \approx 0 \\
\operatorname{erf}(\xi) & \approx 1
\end{aligned}
$$

to derive from Eq. (25)

$$
\left\langle(\Delta x)^{2}\right\rangle_{\mathrm{P}}(t)=2 \kappa_{\mathrm{FL}} v_{\mathrm{A}} t
$$

Obviously, we find a diffusive behavior $\left(\left\langle(\Delta x)^{2}\right\rangle_{\mathrm{P}}(t) \propto t\right)$ for time scales that satisfy $\xi \gg 1$. In this case, we can write

$\left\langle(\Delta x)^{2}\right\rangle_{\mathrm{P}}(t)=2 \kappa_{\perp} t$

with the perpendicular diffusion coefficient of the particle

$\kappa_{\perp}=v_{\mathrm{A}} \kappa_{\mathrm{FL}}$.

According to the results of the last two paragraphs, perpendicular diffusion is recovered in the limit of large time scales. 


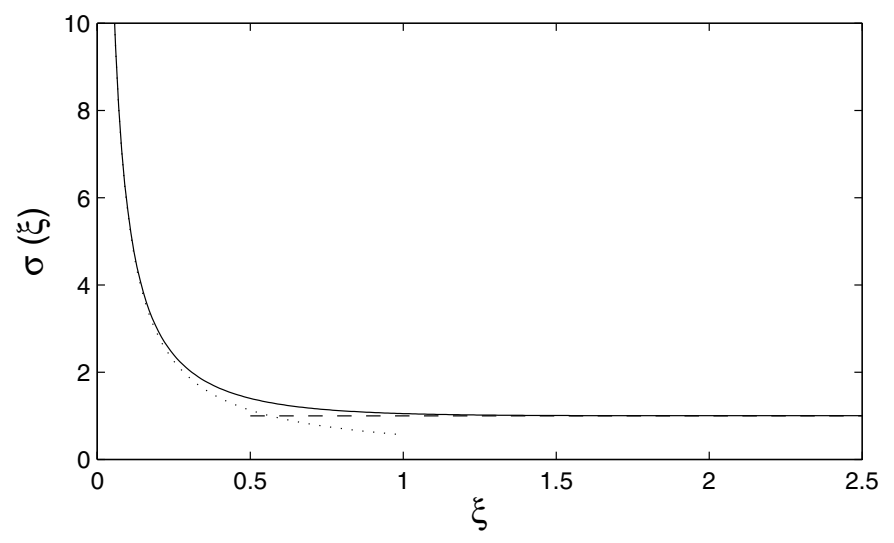

Fig. 1. The time-dependent perpendicular diffusion coefficient $\sigma=$ $\left\langle(\Delta x)^{2}\right\rangle_{\mathrm{P}} /\left(2 \kappa_{\mathrm{FL}} v_{\mathrm{A}} t\right)$ as a function of $\xi=v_{\mathrm{A}} / 2 \sqrt{t / \kappa_{\|}}$. Shown is the result of the numerical integration (solid line) in comparison to the two analytical approximations (dotted line, dashed line).

\subsection{The two characteristic time regimes}

As described in the last two paragraphs, there are two time regimes, namely $\xi \ll 1$ and $\xi \gg 1$. Therefore, we can define a characteristic time scale $t_{\mathrm{c}}$

$\xi\left(t=t_{\mathrm{c}}\right)=1 \quad \Leftrightarrow \quad t_{\mathrm{c}}=\frac{4 \kappa_{\|}}{v_{\mathrm{A}}^{2}}$.

And, hence, we find the two solutions

$\left\langle(\Delta x)^{2}\right\rangle_{\mathrm{P}}(t) \approx\left\{\begin{aligned} 4 \kappa_{\mathrm{FL}} \sqrt{\frac{\kappa_{\|} t}{\pi}} \text { if } t \ll t_{\mathrm{c}} \\ 2 \kappa_{\mathrm{FL}} v_{\mathrm{A}} t \text { if } t \gg t_{\mathrm{c}}\end{aligned}\right.$

for $t \ll t_{\mathrm{c}}$, we find the well known subdiffusive behavior of perpendicular scattering, which can also be found in the magnetostatic limit. For $t \gg t_{\mathrm{c}}$, perpendicular diffusion is recovered. The integral from Eq. (25) can easily be solved numerically. The results are shown in Fig. 1. Clearly, the two different particle transport regimes (subdiffusion and diffusion) can be seen in agreement with our analytical results. For $\xi \ll 1$, we find the well known subdiffusive result, which can also be obtained within the magnetostatic model. For $\xi \gg 1$, however, we find that perpendicular diffusion is recovered due to the plasma wave propagation effects.

\section{Comparison with test-particle simulations}

By using test-particle simulations, several authors (e.g., Qin et al. 2002a,b) numerically explored particle propagation. Qin et al. (2002a) demonstrated that Eq. (31b) is indeed the correct result for perpendicular scattering in the magnetostatic slab model. This agreement between transport theory and simulations confirms the compound transport model (Eq. (18)).

Michałek \& Ostrowski (1996) investigated particle transport in Alfvénic slab turbulence. According to these authors, perpendicular diffusion is recovered. However, very long integration times are required, to achieve recovery of diffusion for perpendicular transport in the slab plasmawave model. This conclusion corresponds to the analytical result derived in this article.

Studies (e.g., Shalchi 2006) have shown time-dependent diffusion coefficients as a function of the dimensionless time $\tau=$

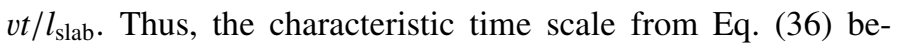
comes

$\tau_{\mathrm{c}}=\frac{v t_{\mathrm{c}}}{l_{\text {slab }}}=\frac{4 v \kappa_{\|}}{v_{\mathrm{A}}^{2} l_{\text {slab }}}=\frac{4}{3} \frac{v^{2}}{v_{\mathrm{A}}^{2}} \frac{\lambda_{\|}}{l_{\text {slab }}}$
Table 1. In this table, the results obtained for the parameters $\alpha$ and $\beta$ obtained by Shalchi \& Kourakis (2007c), adopting the form $\left\langle(\Delta x)^{2}\right\rangle=$ $\alpha z^{\beta}$ for the field line MSD, are shown. In all (but one) cases, we find either superdiffusion $(1<\beta<2)$ or free-streaming $(\beta=2)$ of the field lines. Diffusion $(\beta=1)$ can only be found for $q=0$. The parameter $c_{1}$ is defined as $c_{1}:=\left(\frac{4}{1-q}+\frac{4}{2 v-1}\right)^{-1}$.

\begin{tabular}{lll}
\hline \hline Spectral index $q$ & $\alpha$ & $\beta$ \\
\hline$q=0$ & $2 \kappa_{\mathrm{FL}}$ & 1 \\
$0<q<1$ & $\frac{4 c_{1}}{q(q+1)} l_{\text {slab }}^{1-q} \frac{\delta B_{\text {slab }}^{2}}{B_{0}^{2}} \Gamma(1-q) \sin \left(\frac{\pi q}{2}\right)$ & $q+1$ \\
$1<q<2$ & $\frac{1}{2} \frac{\delta B_{\text {slab }}^{2}}{B_{0}^{2}}$ & 2 \\
\hline
\end{tabular}

where we introduced the parallel mean free path $\lambda_{\|}=3 \kappa_{\|} / v$. For the parameter regimes usually considered, we have $\lambda_{\|} \sim l_{\text {slab }}$ and $v \gg v_{\mathrm{A}}$. Thus, $\tau_{\mathrm{c}}$ is a large number, and the claim that very long integration times are required to achieve recovery of diffusion for perpendicular transport in the slab plasmawave model (as formulated by Michałek \& Ostrowski 1996), can be confirmed theoretically. It should be noted, however, that the wave spectrum used in Michałek \& Ostrowski (1996) is different from that of Eq. (10). Therefore, it is not possible to present a detailed comparison between the result of this article and the simulations of Michałek \& Ostrowski (1996).

\section{Nondiffusive field line random walk and superdiffusion of charged particles}

The results presented in the last section are based on the assumption of diffusive FLRW (see Eqs. (12) and (14)). However, in Shalchi \& Kourakis (2007c), it is demonstrated that, for a different form of the wave spectrum (in comparison to Eq. 10), FLRW behaves superdiffusively. Therefore, in this section, we assume a more general form of the field line MSD

$\left\langle(\Delta x)^{2}\right\rangle_{\mathrm{FL}}=\alpha\left|z \pm v_{\mathrm{A}} t\right|^{\beta}$

where $\beta \geq 1$ and $\alpha>0$. For $\beta=1$ and $\alpha=2 \kappa_{\mathrm{FL}}$, the standard (diffusive) behavior can be recovered. The effect which causes superdiffusive FLRW is related to a steep behavior in the energy range of the wave spectrum. In Table 1 we have shown the values of the parameters $\alpha$ and $\beta$ obtained by Shalchi $\&$ Kourakis (2007c) for different values of the energy range spectral index $q$. As soon as the energy range spectral index exceeds zero $(q>0)$ we find superdiffusion of field lines $(\beta>1)$.

By again applying the compound transport model from Eq. (18), we find

$\left\langle(\Delta x)^{2}\right\rangle_{\mathrm{P}}(t)=\frac{\alpha}{\sqrt{4 \pi t \kappa_{\|}}} \int_{-\infty}^{\infty} \mathrm{d} z\left|z \pm v_{\mathrm{A}} t\right|^{\beta} \mathrm{e}^{-z^{2} /\left(4 t \kappa_{\|}\right)}$.

As demonstrated in Appendix A, the integral from Eq. (40) can be evaluated in terms of the Kummer function $M$, which corresponds to the hypergeometric function ${ }_{1} F_{1}$. In the limits of small time scales (or vanishing Alfvén velocity) and large time scales, we have

$\left\langle(\Delta x)^{2}\right\rangle_{\mathrm{P}} \propto \begin{cases}t^{\beta / 2} & t \ll t_{\mathrm{c}} \\ t^{\beta} & t \gg t_{\mathrm{c}}\end{cases}$

where, again, $t_{\mathrm{c}}$ denotes a characteristic time scale. This is illustrated in Fig. A.1 in Appendix A. 
By assuming that the superdiffusivity is caused by a steep behavior of the wave spectrum in the energy range (see Table 1), we find for $0<q<1$

$\left\langle(\Delta x)^{2}\right\rangle_{\mathrm{P}} \propto \begin{cases}t^{(q+1) / 2} & t \ll t_{\mathrm{c}} \\ t^{(q+1)} & t \gg t_{\mathrm{c}}\end{cases}$

Obviously, we find subdiffusion of charged particles for small time scales and superdiffusion for late times.

\section{Summary and conclusion}

We investigated analytically FLRW and charged particle transport perpendicular to the mean magnetic field in Alfvénic slab turbulence. We demonstrated that, for short time scales, the magnetostatic result (subdiffusion) is still valid. In this regime, plasma wave propagation effects can be neglected. For large times, however, we find the recovery of diffusion. This behavior is in agreement with the results obtained numerically by Michałek \& Ostrowski (1996).

In the magnetostatic or subdiffusive regime, the particle mean square displacement is given by

$\left\langle(\Delta x)^{2}\right\rangle_{\mathrm{P}}\left(t \ll t_{\mathrm{c}}\right) \approx 4 \kappa_{\mathrm{FL}} \sqrt{\frac{\kappa_{\|} t}{\pi}}$,

whereas, in the diffusive regime, we have

$\left\langle(\Delta x)^{2}\right\rangle_{\mathrm{P}}\left(t \gg t_{\mathrm{c}}\right)=2 \kappa_{\perp} t$

with the perpendicular spatial diffusion coefficient

$\kappa_{\perp}=v_{\mathrm{A}} \kappa_{\mathrm{FL}}$.

In this case, the perpendicular mean free path $\lambda_{\perp}$ can be written as

$\lambda_{\perp}=\frac{3}{v} \kappa_{\perp}=3 \frac{v_{\mathrm{A}}}{v} \kappa_{\mathrm{FL}}$

and, with Eq. (13) for the field line diffusion coefficient, we obtain

$\lambda_{\perp}=3 \pi C(v) l_{\text {slab }} \frac{v_{\mathrm{A}}}{v} \frac{\delta B_{\text {slab }}^{2}}{B_{0}^{2}}$.

The characteristic time scale, for which perpendicular transport becomes diffusively, is given by

$\tau_{\mathrm{c}} \approx \frac{4}{3} \frac{v^{2}}{v_{\mathrm{A}}^{2}} \frac{\lambda_{\|}}{l_{\mathrm{slab}}}$

In cases where the particle velocity $v$ is comparable to or smaller than the Alfvén velocity $v_{\mathrm{A}}$, we find diffusion of charged particles for small time scales. This effect could be important for describing heliospheric particle propagation of low energy cosmic rays.

As demonstrated here, plasma wave propagation effects may be important in the case of perpendicular transport of charged cosmic rays. This gives rise to the assumption that other effects such as dynamical turbulence effects or plasma wave damping effects (see Sect. 2) are also important for calculating particle transport parameters. Inclusion of such effects in the calculation of FLRW thus needs to be the suject of future work.

Acknowledgements. This research was supported by the Deutsche Forschungsgemeinschaft (DFG) under the Emmy-Noether Programm (grant SH 93/3-1). We also acknowledge support by the DFG grant Schl 201/17-1. As a member of the Junges Kolleg, A. Shalchi also acknowledges support by the Nordrhein-Westfälische Akademie der Wissenschaften.

\section{Appendix A: General mean square deviation}

By assuming a more general form of the field line MSD which also allows for superdiffusive behavior (as demonstrated in Sect. 6), we obtain the following form for the mean square displacement of the particles

$\left\langle(\Delta x)^{2}\right\rangle_{\mathrm{P}}(t)=\frac{\alpha}{\sqrt{4 \pi t \kappa_{\|}}} \int_{-\infty}^{\infty} \mathrm{d} z\left|z \pm v_{\mathrm{A}} t\right|^{\beta} \mathrm{e}^{-z^{2} /\left(4 t \kappa_{\|}\right)}$.

This integral can be evaluated in terms of the Kummer function $M\left(a, b, \xi^{2}\right)$ or hypergeometric function ${ }_{1} F_{1}$ (e.g., Abramowitz \& Stegun 1974; Gradshteyn \& Ryzhik 2000) and yields

$$
\begin{aligned}
\left\langle(\Delta x)^{2}\right\rangle_{\mathrm{P}}= & \frac{\alpha \mathrm{e}^{-\xi^{2}}\left(4 t \kappa_{\|}\right)^{(\beta-1) / 2}}{2 \sqrt{\pi}}\left\{\beta v_{\mathrm{A}} t \Gamma\left(\frac{\beta}{2}\right)\right. \\
& \times\left[M\left(\frac{1-\beta}{2}, \frac{3}{2},-\xi^{2}\right) \mathrm{e}^{\xi^{2}}-M\left(\frac{2+\beta}{2}, \frac{3}{2}, \xi^{2}\right)\right] \\
& +\sqrt{4 t \kappa_{\|}} \Gamma\left(\frac{1+\beta}{2}\right)\left[M\left(-\frac{\beta}{2}, \frac{1}{2},-\xi^{2}\right) \mathrm{e}^{\xi^{2}}\right. \\
& \left.\left.+M\left(\frac{1+\beta}{2}, \frac{1}{2}, \xi^{2}\right)\right]\right\}
\end{aligned}
$$

In turn, we consider the two limiting cases of $\xi \ll 1$ and $\xi \gg 1$.

\section{A.1. The limit $\xi \ll 1$}

In the limit $\xi \ll 1$, which corresponds to either small time scales, or vanishing Alfvén speed, $v_{\mathrm{A}} \rightarrow 0$, a Taylor expansion of the Kummer function $M\left(a, b, \xi^{2}\right)$ yields (Abramowitz \& Stegun 1974)

$M\left(a, b, \xi^{2}\right)=1+\frac{a}{b} \xi^{2}+\ldots$

Therefore, we obtain for the particle mean square displacement

$$
\begin{aligned}
\left\langle(\Delta x)^{2}\right\rangle_{\mathrm{P}}(t) & =\frac{\alpha\left(4 t \kappa_{\|}\right)^{\beta / 2}}{\sqrt{\pi}} \Gamma\left(\frac{\beta+1}{2}\right)\left[1+\beta \xi^{2}\right] \\
& \approx \frac{\alpha\left(4 t \kappa_{\|}\right)^{\beta / 2}}{\sqrt{\pi}} \Gamma\left(\frac{\beta+1}{2}\right)
\end{aligned}
$$

which is proportional to $t^{\beta / 2}$ and, regarding the temporal behavior, agrees with Eq. (31b) if $\alpha=2 \kappa_{\mathrm{FL}}$ and $\beta=1$.

\section{A.2. The limit $\xi \gg 1$}

In this limit, which corresponds to the limit of large time scales, we can use the asymptotic behavior of the Kummer function. To lowest order, we have for example (Abramowitz \& Stegun 1974; and Gradshteyn \& Ryzhik 2000)

$$
\begin{gathered}
M\left(\frac{1-\beta}{2}, \frac{3}{2},-\xi^{2}\right) \propto \xi^{\beta-1} \\
M\left(-\frac{\beta}{2}, \frac{1}{2},-\xi^{2}\right) \propto \xi^{\beta}
\end{gathered}
$$

whereas the contributions of the other Kummer functions in Eq. (A.2) are exponentially damped. This gives rise to asymptotic behavior of $\left\langle(\Delta x)^{2}\right\rangle \propto t^{\beta}$, which, for $\beta=1$, yields diffusive 


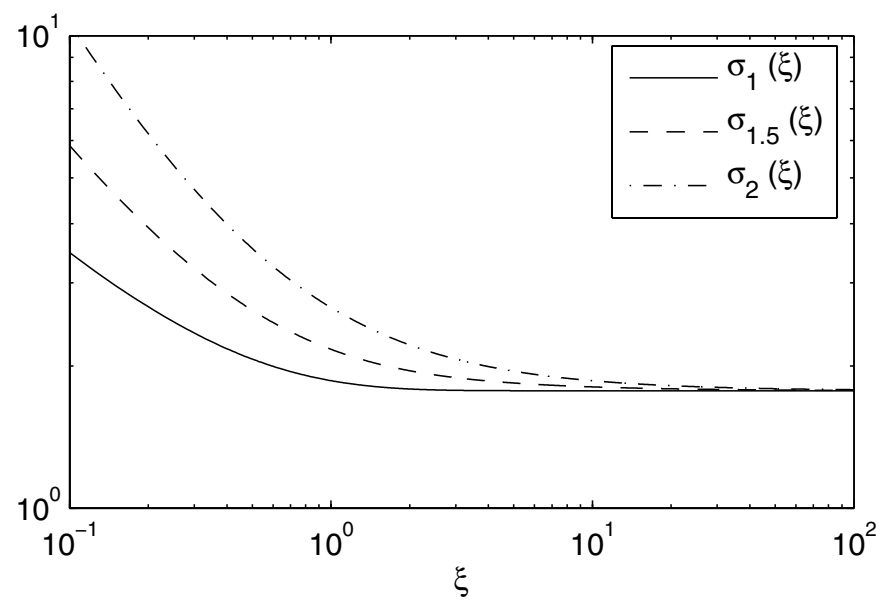

Fig. A.1. The time-dependent perpendicular diffusion coefficient $\sigma_{\beta}$ as defined in Eq. (A.6) as a function of $\xi=v_{\mathrm{A}} / 2 \sqrt{t / \kappa_{\|}}$. Shown are the values of $\sigma$ for $\beta=1$ (solid line), $\beta=1.5$ (dashed line), and $\beta=2$ (dash-dot line). For $\xi \ll 1$ and for $\xi \gg 1$, we again find the subdiffusive and diffusive results, respectively, which we had obtained for $\beta=1$ (Eq. (25)). Thus, our considerations from Sects. A.1 and A.2 are confirmed.

behavior (proportional to $t$ ), whereas, for $\beta>1$, this results in superdiffusive behavior. To test this result, the function

$\sigma_{\beta}(\xi) \equiv \frac{\left\langle(\Delta x)^{2}\right\rangle_{\mathrm{P}}}{\left(2 \kappa_{\mathrm{FL}} v_{\mathrm{A}} t\right)^{\beta}}$ (with the exact mean square displacement from Eq. (A.2)) is shown in Fig. A.1 as a function of $\xi$ for different values of $\beta$. Obviously, an asymptotic behavior $t^{\beta}$ can be confirmed.

\section{References}

Abramowitz, M., \& Stegun, I. A. 1974, Handbook of Mathematical Functions (New York: Dover Publications)

Bieber, J. W., Matthaeus, W. H., Smith, C. W., et al. 1994, ApJ, 420, 294

Gradshteyn, I. S., \& Ryzhik, I. M. 2000, Table of integrals, series, and products (New York: Academic Press)

Jokipii, J. R. 1966, ApJ, 146, 480

Kóta, J., \& Jokipii, J. R. 2000, ApJ, 531, 1067

Matthaeus, W. H., Gray, P. C., Pontius, D. H. Jr., \& Bieber, J. W. 1995, Phys. Rev. Lett., 75, 2136

Michałek, G., \& Ostrowski, M. 1996, Nonlin. Proc. Geophys., 3, 66

Qin, G., Matthaeus, W. H., \& Bieber, J. W. 2002a, Geophys. Res. Lett., 29, 1048 Qin, G., Matthaeus, W. H., \& Bieber, J. W. 2002b, ApJ, 578, L117

Schlickeiser, R. 2002, Cosmic Ray Astrophysics (Berlin: Springer-Verlag)

Schlickeiser, R., \& Achatz, U. 1993, J. Plasma Phys., 49, 63

Shalchi, A. 2006, A\&A, 453, L43

Shalchi, A., \& Kourakis, I. 2007a, Phys. Plasmas, 14, 9

Shalchi, A., \& Kourakis, I. 2007b, J. Phys. A: Math. Theor., 40, 11191

Shalchi, A., \& Kourakis, I. 2007c, Phys. Plasmas, in press

Webb, G. M., Zank, G. P., Kaghashvili, E. K., \& le Roux, J. A. 2006, ApJ, 651, 211 